\title{
EDITORIAL
}

Torsten Braun

Universität Bern

\section{Das Internet der Zukunft}

\section{Vom ARPANET über das World Wide Web zum Internet der Dinge}

Im Jahr 2009 konnte das Internet seinen 40. Geburtstag feiern, da man allgemein das Geburtsjahr des Internet mit der Inbetriebnahme des ARPANET im Jahr 1969 verbindet. Hauptzweck war damals die Verbindung von mehreren Großrechnern, die räumlich voneinander entfernt standen. Das Internet entwickelte sich maßgeblich auf der Basis des ARPANET. Während das Internet in den 1970er und 1980er Jahren fast ausschließlich in der akademischen Forschung benutzt wurde, änderte sich dies schlagartig mit der Entwicklung des World Wide Web (WWW) im Jahr 1989. Das WWW ermöglichte mit Hilfe von auf persönlichen Computern laufenden Browsern auch der breiten Allgemeinheit ohne spezielle Fachkenntnisse, Informationen von Web-Servern abzurufen. Im Lauf der Zeit entwickelten sich eine Vielzahl von Diensten und Anwendungen für geschäftliche und private Zwecke. Das Internet stellt heute eine unverzichtbare Infrastruktur dar, welche mittlerweile ähnlich wichtig ist wie andere Infrastrukturen, z. B. für Verkehr und Stromversorgung. Insbesondere hat sich das Internet zu einer universalen Kommunikationsinfrastruktur entwickelt, über die nicht mehr nur Emails, Dateien oder Web-Seiten übertragen werden, sondern über die auch (mobil) telefoniert wird oder die zur Verteilung von Fernsehsendungen dient. Die historische Notiz dieses Heftes fasst die Entwicklung des Internet in den letzten 40 Jahren zusammen.

Bis heute werden über das Internet meistens Computer miteinander verbunden. Jedoch ist aufgrund der weiter fortschreitenden Miniaturisierung eine zunehmende Integration von elektronischen Komponenten und Kleinstcomputern in Alltagsgegenstände bzw. Dinge zu beobachten. Werden diese Dinge bzw. ihre integrierten Computer mit dem Internet verbunden, z. B. um physikalische Größen zu messen oder Prozesse zu steuern, spricht man häufig vom „Internet der Dinge“. Der Beitrag von Friedemann Mattern und Christian Flörkemeier (ETH Zürich) erläutert diesen Begriff, führt in die technologischen Anforderungen und Lösungsansätze ein, stellt Beispielanwendungen vor und diskutiert gesellschaftliche Fragestellungen, die sich aus dieser Entwicklung ergeben.

\section{Internet-Forschung im nationalen und internationalen Umfeld}

Die Nutzung des Internet hat sich in den letzten vier Jahrzehnten sehr stark verändert. Die Entwickler der ersten, im Internet eingesetzten Kommunikationsprotokolle konnten diese Entwicklung nicht voraussehen. Daher ist es nicht überraschend, dass die heutigen Protokolle, auf denen das Internet basiert, viele Anforderungen, welche heutige Anwendungsfelder an das Internet stellen, nicht mehr oder nur noch unzureichend erfüllen können. In diesem Zusammenhang seien Sicherheit, Benutzermobilität oder höchste Zuverlässigkeit genannt. In der Vergangenheit versuchte man neue Anforderungen und Funktionen in der Regel durch Optionen und Erweiterungen existierender Protokolle oder durch die Einführung zusätzlicher Protokolle zu unterstützen. Allerdings ist es sehr schwer, Protokolle wie das Internet Protocol (IP) oder das Transmission Control Protocol (TCP) aufgrund ihrer globalen Verbreitung zu ersetzen. Beispielsweise wird seit den frühen 1990er Jahren versucht, die Version 4 von IP durch die Version 6 abzulösen, was bisher in den meisten Teilen des Internet nicht gelungen ist. So basiert das heutige Internet immer noch auf Protokollen, die in den 1970er Jahren entstanden sind. Innerhalb der Internet-Forschungs-Community wird heute intensiv diskutiert, wie die Architektur des zukünftigen Internet, d. h. in 10 bis 20 Jahren aussehen sollte, um die neuen Anforderungen erfüllen zu können. Ist es möglich mit einer evolutionären Entwicklung das Internet der Zukunft zu 


\section{$\{$ EDITORIAL}

gestalten oder sollte man eher eine völlig neue Architektur, welche die aktuelle Internet-Architektur vollständig ablöst und ersetzt, für das Internet der Zukunft entwickeln? Diese Fragestellungen beschäftigen Forscher aktuell weltweit. Um die Internet-Forschung voranzutreiben, sind nicht nur theoretische Überlegungen notwendig, sondern neue und innovative Konzepte und Methoden müssen ihre Einsetzbarkeit in realen Umgebungen unter Beweis stellen. Aus diesem Grund gibt es eine große Anzahl von internationalen Programmen, welche Experimentierplattformen für die Forschung entwickeln und bereitstellen. Der Artikel von Stefan Fischer (Universität zu Lübeck) und Paul Müller (TU Kaiserslautern) beschreibt die Anstrengungen, die hinsichtlich des Aufbaus von Infrastrukturen zur experimentellen Erforschung neuer Konzepte und Mechanismen für das zukünftige Internet vor allem in Deutschland und Europa verfolgt werden.

\section{Anforderungen an das Internet der Zukunft}

Eine Vielzahl von Forschern beschäftigt sich weltweit mit der Frage, wie Architekturen, Protokolle, Mechanismen und Konzepte für das zukünftige Internet aussehen sollten. Im Frühjahr 2009 organisierten Georg Carle (TU München), David Hutchison (Lancaster University), Bernhard Plattner (ETH Zürich) und James Sterbenz (University of Kansas, Lawrence) auf Schloss Dagstuhl einen Perspektiven-Workshop mit dem Titel "Architecture and Design of the Future Internet". Gerade aus dem deutschsprachigen Raum kamen viele interessante Beiträge, die sich mit aktuellen Herausforderungen und Problemen des Internet sowie Lösungsansätzen beschäftigen. Insbesondere werden diese Forschungsarbeiten in etlichen europäischen Verbundprojekten durchgeführt. Dies zeigt auch, dass die InternetForschung im deutschsprachigen Raum sehr gut etabliert ist und international Anerkennung findet. Aus dem Kreis der Workshop-Teilnehmer konnten Autoren gewonnen werden, welche aktuelle Herausforderungen des zukünftigen Internet betrachten und in diesem Themenheft ihre aktuellen Forschungsaktivitäten im Bereich des Future Internet vorstellen.

Wesentliche Aspekte bei der Entwicklung einer für die Zukunft geeigneten Internet-Architektur sind natürlich Robustheit, Zuverlässigkeit und Verfügbarkeit des Internet als globale Kommunikationsinfrastruktur. Der Artikel von Nils Kammenhuber, Ali Fessi und Georg Carle (TU München) beschreibt Protokolle und Techniken auf verschiedenen Schichten, welche versuchen, die Widerstandsfähigkeit des Internets gegenüber Störungen, z. B. verursacht durch Fehler, Überlast oder auch Sicherheitsangriffen, zu erhöhen.

Gerade im letzten Jahrzehnt hat sich die mobile Kommunikation sehr stark verbreitet. Das Internet als globales Kommunikationsnetz muss daher auch die Mobilität von Benutzern unterstützen, und zukünftig auch die Mobilität von Dingen. Dies bedeutet auch, dass die Kommunikation bei sich schnell bewegenden Menschen und Dingen möglichst unterbrechungsfrei gewährleistet sein sollte. Karin Hummel, Andrea Hess und Harald Meyer (Universität Wien) diskutieren die Herausforderungen von Geräte- und Ressourcenmobilität im Internet der Zukunft.

Das Management eines globalen Netzes wie dem Internet stellt für die Netzbetreiber eine hoch komplexe Aufgabe dar, speziell da sich das Internet aus vielen kleineren, durch unabhängige Betreiber verwalteten Teilnetzen zusammensetzt. Neben den Netzbetreibern kommen als weitere Akteure Dienstanbieter und Nutzer hinzu, welche teilweise gegensätzliche Interessen verfolgen. Der Artikel von Tanja Zseby, Michael Kleis und Thomas Hirsch (Fraunhofer Institut FOKUS, Berlin) beschreibt wie Kooperationsmechanismen zwischen den verschiedenen Akteuren das Netzmanagement und die Qualität von Diensten im Internet unterstützen können.

Die reibungslose Einführung neuer Protokolle und Technologien gestaltet sich im Internet als sehr schwierig, da es kaum möglich ist, zu einem vorbestimmten Zeitpunkt in allen End- und Zwischensystemen bestimmte Protokolle oder Mechanismen durch andere vollständig zu ersetzen. Overlay-Netze haben sich dabei als ein vielversprechendes Konzept erwiesen, um neue Funktionen schrittweise im Internet einzuführen. Oliver Waldhorst, Roland Bless und Martina Zitterbart (Karlsruher Institut für Technologie) beschreiben die Funktionsweise von Overlay-Netzen und stellen den eigenen Ansatz der Spontanen Virtuellen Netze (SpoVNet) vor. Anhand mehrerer konkreter Beispiele wird gezeigt, wie mit Hilfe von SpoVNet neue Funktionen im Internet graduell eingeführt werden können.

Das Thema der virtuellen Netze, d.h. der Anwendung von Virtualisierungsmethoden im Internet, vertieft der Beitrag von Andreas Berl, Andreas Fischer und Hermann de Meer (Universität Passau). Es wird erläutert, wie Hostund Netzwerkressourcen virtualisiert werden können und wie sich mit Hilfe von Virtualisierung flexible und rekon- 
figurierbare Netze realisieren lassen. Weitere Vorteile von Netzwerk-Virtualisierung liegen in der Unterstützung von Netzmanagement, der erhöhten Robustheit und der verbesserten Energieeffizienz zukünftiger Netze.

\section{Torsten Braun}

\section{Danksagung}

Ein großer Dank geht an die Autoren, die sich bereit erklärt haben, spannende und aktuelle Beiträge zu diesem Themenheft zu erstellen. Des Weiteren haben zahlreiche Gutachter die eingereichten Artikel sehr gründlich begutachtet und mit ihren Kommentaren und Verbesserungsvorschlägen zur hohen Qualität der Beiträge und damit dieses Themenhefts beigetragen. In diesem Zusammenhang sei folgenden Personen für Ihr Engagement herzlichst gedankt: Marcus Brunner, Kirsten Dolfus, Anna Förster, Robert Henjes, Wolfgang Kellerer, Peter Kropf, Simon Leinen, Martin Mauve, Michael Menth, Dennis Pfisterer, Günter Schäfer, Otto Spaniol, Burkhard Stiller, Kurt Tutschku und Thiemo Voigt. 